\title{
CONTOUR SIMULATIONS OF ASTROPHYSICAL JETS
}

\author{
L. ZANINETTI \\ Istituto di Fisica Generale \\ Via Pietro Giuria 1 \\ 10125 Torino, Italy
}

ABSTRACT. We apply the theory of the Kelvin-Helmholtz instabilities to trace the jet's pattern. A certain level of noise is introduced.

Adopted parameters: $m$ is the azimuthal number chosen, $n$ the number of the e-folding lengths after which the jet becomes unstable, $M$ the Mach number, $v_{0}$ the density contrast, $L$ the jet's length, $a_{0}$ the initial amplitude, "scaling" represents the ratio between the length of the $x$-axis of the observed map and the jet's length, $\theta$ is the opening angle, and $\alpha$ the angle of rotation from the horizontal position. In order to make more realistic simulations we introduce some noise; the blobs will be placed at a position given by a pseudo-random real number taken from a normal (Gaussian) distribution with mean $\bar{x}$ where $\bar{x}$ is the predicted distance, and standard deviation $\sigma$ where $\sigma=\lambda /$ noise.

The relativistic particles are assumed to diffuse away from the accelerating regions (blobs) of increasing radius $r_{0}$, through a steady random walk of mean free path $\lambda$. We then take a grid of 100 by 100 points on the plane of the trajectory selecting in each one the maximum intensity from the various blobs. We report in Fig. 1 the isoemissivity contours in arbitrary units of the initial flux $F_{0}$. "zincr" is the distance between the various levels.

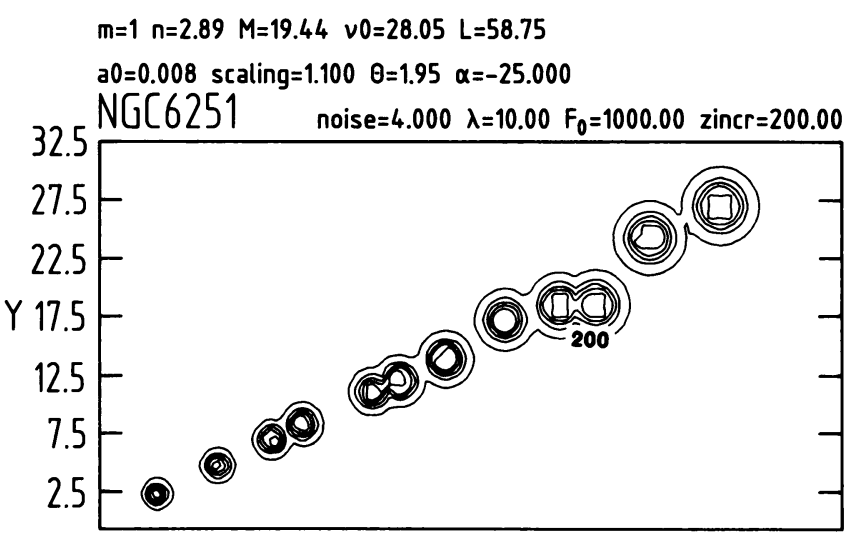

